\title{
Inspection of the Leakage for the Closure Plug of Heavy Water Reactor using Infrared Thermography in Nuclear On-site Application
}

by S. G. Lee*and W. T. Kim**'

\author{
*Nuclear Power Laboratory, Korea Electric Power Research Institute, Daejeon, Korea (South) 305-760, \\ sglee@kepri.re.kr \\ ${ }^{\star \star}$ Div. of Mechanical and Automotive Engineering, Kongju National University, Cheonan (Chungnam), Korea (South) \\ 331-717, kwt@kongju.ac.kr ( ${ }^{\text {, }}$ corresponding author)
}

\begin{abstract}
In this work, infrared thermography was applied for the early detection and condition monitoring on the leakage of heavy water caused at closure plug during on-site operation of the nuclear reactor. In order to monitor the temperature distributions coincided with the leakage of heavy water led out of the closure plug for each fuel channels, infrared measuring technique was reviewed. From the work of this study, it was concluded that the location of leakage could be easily found and the leakage status during its on-site operation was monitored in real time. As results, it was found that infrared measuring technique can be proposed as the method of condition monitoring at on-site operation of nuclear reactor and be contributes to improve the accuracy of nuclear facilities
\end{abstract}

\section{Introduction}

Over the past decades, there have been several ways to monitor the leakage testing of the closure plug at heavy water reactor in nuclear plant during its on-site application. As the industries are highly developed, the rate of energy usage is explosively increasing. However, the amount of fuel energy like coil and oil deposits as energy sources is unfortunately limited and the price of oil as most of energy source is continuously increasing. On the other hand, since gas emission by using these fuel energy are the main culprit of air pollution and provides the global warming by causing the increment of $\mathrm{CO}_{2}$, the demand of nuclear energy are proportionally rising up by its advantages in relatively low cost and no gas emission. However, people consider the safety of nuclear plant at both sides of radioactive contamination and nuclear safety during its on-site operation. For the case of nuclear safety during its on-site operation, the program of predictive maintenance for nuclear facilities to monitor the operation of nuclear reactor should be very credential. In this work, infrared thermography was applied for the early detection and condition monitoring on the leakage of heavy water caused at closure plug during onsite operation of the nuclear reactor.

\section{Background of the study}

Until now, there has been no precise monitoring measurement facility to follow up the condition monitoring for the closure and to track the leakage location at all fuel channels when the tritium concentration of nuclear reactor is increasing by the leakage of heavy water.
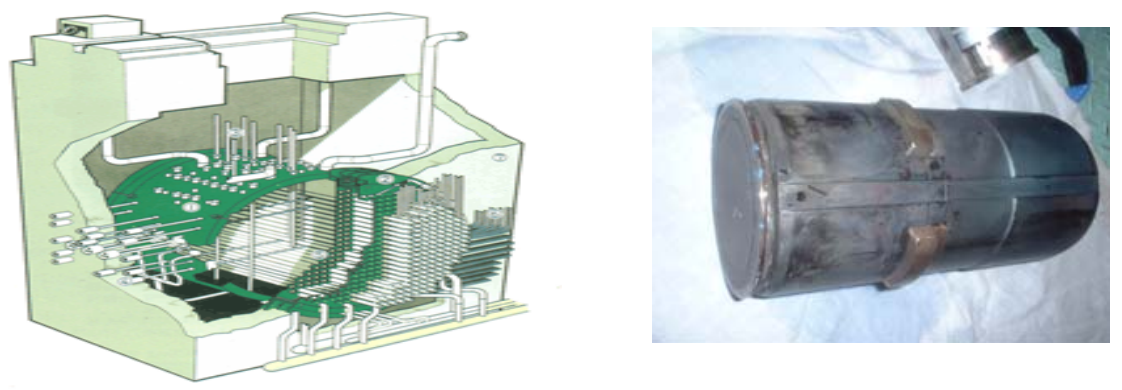

(a) Structure of heavy water reactor (b) Photography of closure plug

Fig. 1 Structure and closure plug considered in the study 
As further study, following Fig. 1 shows the the leakge detection system of the closure plug at heavy water reactor in nuclear plant. In case of testing method using CCTV camera, the precision degree of the testing is considerably low. At present, in order to measure the leakage of heavy water at the closure plug during on-site operation of nuclear reactor, there are two testing methods; one is using the CCTV camera and another one is using the fuelling machine, it consumes much time for the testing and, above all, it brings about the increase cause of the tritium concentration in testing period. Therefore, even though these two methods have been used as detection ones, each of them has fatally own constraints. Also,

\section{Experimental work}

In order to monitor the temperature distributions coincided with the leakage of heavy water led out of the closure plug for each fuel channels, the temperature contours including hot spts acquired by infrared measuring technique was analyzed. Fig. 2 (a) show the experimental set-up for the condition monitoring in the heavy water reactors and Fig. 2 (b) shows the location of detection to be diagnosed from infrared thermography.

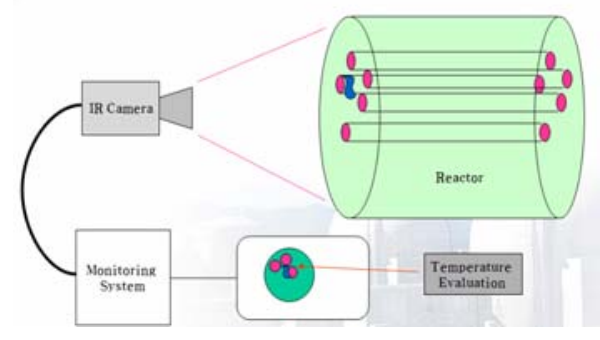

(a) The system of leakage testing

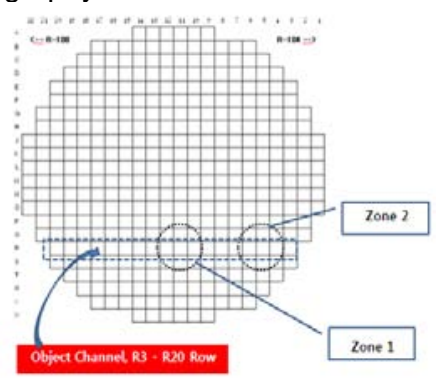

(b) The location to be monitored

Fig. 2 Schematic diagram of experimental apparatus

\section{Results and discussions}

All figures of Fig. 3 showed imaging's for zone-1 and zone-2, respectively, of infrared thermography for detecting the leakage in the front of the closure plug channel in heavy water reactors in the nuclear plants.

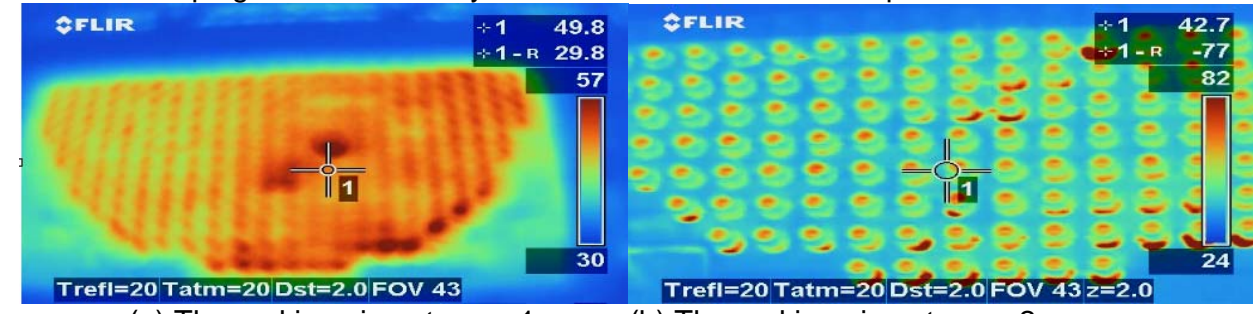

(a) Thermal imaging at zone-1

Fig. 3. Infrared imaging from zone 1 of closure plug $(\varepsilon=0.96)$

\section{Conclusions}

From infrared thermography, the thermal imaging for detecting the leakage of heavy water from the closure plug in the nuclear plants was evaluated on-site. Since there were much radioactivity, condition monitoring using thermography was very useful to observe the temperature distributions without risks and concluded as one enough efficient to monitor.

\section{REFERENCES}

[1] G. J . Dau, "A Review of O nline Leak Detection Methods for Reactor System", 3rd Conference on Periodic Inspection of Pressurized Components. I. Mech. E. J ondon, p. 67-73, 1976

[2] D. O. Harris, et al. Electric Power Research Institute Report, EPRI CS-1896, EPRI, Palo Alto, CA, p. 124-1, 1980

[3] S. G. Lee, et al. "Tritium and Coolant Leak Evaluation for Fuel Channel Closure Plug in Heavy Water Reactor Using Multiple Acoustic Sensors", Transactions of the Korean Nuclear Society Autumn Meeting, Pyeongchang, Korea, p. 19-25, 2008 